\title{
Effect of Energy Substrate Dynamics on Mitochondrial Activity and Oxidative Stress Levels of in vitro Maturing Bovine Oocytes
}

\author{
Zulaiha A. Rahman ${ }^{1}$, Siti Fatimah Ibrahim ${ }^{1}$, Nurul Atikah Osman ${ }^{1}$, Farah Hanan Fathihah Jaffar ${ }^{1}$ \\ \& Khairul Osman ${ }^{2}$ \\ ${ }^{1}$ Department of Physiology, Faculty of Medicine, Universiti Kebangsaan Malaysia, Kuala Lumpur, Malaysia \\ ${ }^{2}$ Faculty of Health Sciences, Universiti Kebangsaan Malaysia, Kuala Lumpur, Malaysia \\ Correspondence: Siti Fatimah Ibrahim, Department of Physiology, Faculty of Medicine, Universiti Kebangsaan \\ Malaysia, Jalan Yaacob Latif, Cheras, Kuala Lumpur 56000, Malaysia. Tel: 60-391-458-615. Fax: \\ 603-2693-9687. E-mail: timi@ukm.edu.my
}

Received: October 3, 2017

Accepted: December 15, 2017

Online Published: December 31, 2017

doi:10.5539/jas.v9n13p14

URL: https://doi.org/10.5539/jas.v9n13p14

\begin{abstract}
This study was conducted to explore the effect of energy substrates in the culture medium during in vitro maturation of bovine oocytes. A modified TCM199 medium (M-7528) was used to mature bovine oocytes in vitro. Oocytes were supplemented with different pyruvate $(0.1,0.2,0.4 \mathrm{mM})$ and glucose $(1.5,5.6,20.0 \mathrm{mM})$ concentrations for 48 hours at $38.5^{\circ} \mathrm{C}$ under $5 \% \mathrm{CO}_{2}$ atmosphere with $95 \%$ humidity. Their maturity was checked at 24 and 48 hours. After 48 hours, the denuded oocytes were stained with fluorescent dye JC-1 and avidin-FITC. Fluorescent dye JC-1 is a membrane permeable to the cell and would indicates membrane activity or its organization. Fluorescence intensity of avidin-FITC determination using corrected total cell fluorescence (CTCF) expressed oxidative stress level. There is a significant contribution of energy substrates towards oocyte maturation. Pyruvate at $0.2 \mathrm{mM}$ produced mature oocytes with a diameter of $\geq 120 \mu \mathrm{m}$, promoted oocytes maturation to metaphase II (MII) stage faster and reduced cell's oxidative stress levels. In comparison, $5.6 \mathrm{mM}$ glucose is the optimum concentration for glucose to reduce cell stress level. Unfortunately, this concentration only produced mature oocytes with a small diameter of up to $116 \mu \mathrm{m}$. All changes were significant at the level of $\mathrm{p}<0.05$. As a conclusion, pyruvate at $0.2 \mathrm{mM}$ is the optimum concentration for in vitro maturation after taking cell's stress level into consideration.
\end{abstract}

Keywords: oocyte, bovine, in vitro maturation, mitochondrial organization, JC-1, 8-OHdG

\section{Introduction}

Recent growing interest in in vitro production is reflected by the extensive efforts that have been made to improve the in vitro systems for oocyte maturation and fertilization. Despite recent developments in the in vitro maturation (IVM) and in vitro fertilization (IVF), it is recognized that the developmental capacity of bovine IVM oocytes is inferior to that of the in vivo matured oocytes (Sirad \& Blondin 1996). The low developmental competence of IVM oocytes is related to their quality at the beginning of maturation. Thus, the oocytes quality before IVM is the most important factor that determines the success of IVF and in vitro development of embryos.

Cumulus-oocyte complexes (COCs) are routinely selected for IVM on the basis of the assessment of morphological features such as cumulus thickness and compactness, number of cumulus cell layers surrounding the oocytes and the homogeneity of ooplasm (Han et al., 2006; Ishizaki, Watanabe, Bhuiyan, \& Fukui, 2009). Moreover, oocyte quality can be assessed by several methods including size and metabolic activity (Ebner, Moser, Sommergruber, \& Tews, 2003; Pawlak, Renska, Pers-Kamczyc, Warzych, \& Lechniak, 2011). With regards to oocyte size, some of the bovine oocytes acquired full meiotic competence when reaching a diameter of $100-115 \mu \mathrm{m}$. Oocytes with a diameter larger than $120 \mu \mathrm{m}$ are commonly classified as fully competent to be fertilized successfully (Hunter 2000; Anguita, Jimenez, Izquierdo, Mogas, \& Paramio, 2007; Pawlak, Renska, Pers-Kamczyc, Warzych, \& Lechniak, 2011). Therefore, it is assumed that an oocyte displaying both good morphological features of COCs and a diameter $\geq 120 \mu \mathrm{m}$ is categorized as good quality oocyte.

In any culture medium, energy substrate is an important ingredient for the optimum in vitro development of embryos (Herrick et al., 2006; Tsuji et al., 2009). Among the most researched energy substrate are glucose and 
pyruvate (Nandi, Kumar, Manjunatha, Ramesh, \& Gupta, 2008). Glucose helps cumulus-oocyte-complex (COCs) to mature (Pries \& Gardner, 2005), and is essential for cellular functions during oocyte maturation (Sutton-McDowall, Gilchrist, \& Thompson, 2010). Pyruvate is a main energy substrate used directly for oocyte maturation and resumption of meiosis (Biggers, Whittingham, \& Donahue, 1967; Roberts et al., 2004; Pries, Seidel, \& Gardner, 2005; Sutton-McDowall, Gilchrist, \& Thompson, 2010). This metabolic activity plays a crucial role in oocyte quality because glycolytic activity in mature oocytes is correlated with increased embryo development (Krisher, 2004). Thus, level of both substrates can have a profound effect on oocyte maturation and adequate levels of glucose and pyruvate are important for progression of meiosis to metaphase II. Hence, understanding energy substrate metabolism of the oocyte throughout IVM may aid in optimizing maturation condition.

Mitochondria are double membrane organelles that play a fundamental role in the cell and mitochondrial dysfunction. It has been linked with several pathologies, including infertility (Wallace, 1999; Ramalho-Santos et al., 2009). Mitochondria's vital role in the oocytes metabolism is to provide ATP for fertilization and preimplantation embryo development. The rate of oocyte respiration depends on two major factors: (i) the efficiency of conversion in the oocyte cytoplasm of metabolic precursors such as glucose to pyruvate, and (ii) the efficiency of the mitochondria matrix in the conversion of pyruvate to ATP (Wilding et al., 2001; Vellila, Gonzalez, Vidal, Izquierdo, \& Panamio, 2006; Ramalho-Santos et al., 2009). JC-1 (5,5'6,6'-tetrachloro1,1 '3,3'tetraethylbenzimidazolycarbo cyanine iodide) is a fluorescent dye which accumulates in mitochondria and one of the most specific stains currently used to detect mitochondrial membrane potential for oocytes (Smiley et al., 1991; Wilding et al., 2001; Blerkom \& Davis, 2007; Thompson, Lane, \& Gilchrist, 2007). Due to this, JC-1 can be used as an indicator of mitochondrial activity.

It is estimated that $85-90 \%$ of a cell's oxygen is consumed by mitochondria in oxidative phosphorylation. Due to high usage of oxygen, potentially harmful reactive oxygen species (ROS) such as superoxide radicals, hydroxyl radicals and hydrogen peroxide are generated in abundance (Sugino, 2005, 2006; Ramalho-Santos et al., 2009). Past studies have indicated that mitochondria are the major ROS generator, with $0.2-2 \%$ of the oxygen taken up by the cell is converted to ROS (Ramalho-Santos et al., 2009).

Oxidative stress occurs as a consequence of the excessive production of reactive oxygen species and impaired of antioxidant defence mechanism. According to Sugino (2005), ROS in oocytes are produced within the follicle, especially during the ovulatory process. Studies have indicated that oxidative stimulation plays an important physiological role in maturation in which it promotes oocyte maturation and follicular wall rupture within the follicle. However, when unregulated the excessive production of ROS may lead to an increased risk of poor oocyte quality (Tamura et al., 2007; Ramalho-Santos et al., 2009). Hence, the present study would like to determine the relationship between oxidative stress level and mitochondrial activity in matured oocytes. Intrafollicular concentrations of 8-hydroxy-2'-deoxyguanosine (8-OHdG) were used as a sensitive indicator of DNA damage. As a biomarker of oxidative stress, 8-OHdG was measured through the use of avidin conjugated-fluorescein isothiocyanate (FITC) (Struthers, Patel, Clark, \& Thomas, 1998).

In summary this study was designed to: (1) determine if COCs diameter is a good reflection of oocyte maturation;

(2) determine the optimum glucose and pyruvate concentration for mitochondrial activity in matured oocytes; and

(3) determine the optimum glucose and pyruvate concentration for oxidative stress levels in matured oocytes.

\section{Material and Methods}

\subsection{Oocyte Collection}

Bovine ovaries were obtained at a local abattoir and transported to the laboratory in sterile $0.01 \mathrm{M}$ Phosphate-Buffered Saline (PBS), pH 7.4 at $37{ }^{\circ} \mathrm{C}$. Transportation was done within 3 hours after sacrifice. After the ovaries were washed twice in sterilized PBS solution, cumulus-oocyte complexes (COCs) were obtained from the ovaries by a slicing method. COCs having four to five layers of intact cumulus cells and homogeneous cytoplasm were then rinsed thrice in PBS medium containing 5\% (v/v) calf serum, 10,000 IU Penicillin and $10 \mathrm{mg}$ of Streptomycin. They were then used for later experiment.

\subsection{In vitro Maturation}

The IVM medium used was a tissue culture medium 199 (with 25 mM HEPES, Earl's salts, L-glutamine and 2 mg $\mathrm{mL}^{-1}$ sodium bicarbonate; Sigma) modified by the addition of $4 \mathrm{mg} \mathrm{mL}^{-1}$ bovine serum albumin and gentamicin 50 $\mu \mathrm{g} \mathrm{mL}{ }^{-1}$. Each 10 to 20 oocytes were then cultured in $100 \mu \mathrm{L}$ of maturation solution under mineral oil. Ten oocytes per mineral oil drop was incubated for 48 hours at $38.5^{\circ} \mathrm{C}$ under $5 \% \mathrm{CO}_{2}$ atmosphere with $95 \%$ humidity. Oocytes 
were checked for maturity at 24 and 48 hours. After 48 hours, oocytes were denuded mechanically from cumulus cells in modified phosphate-buffered saline supplemented with $1 \mathrm{mg} / \mathrm{ml}$ hyaluronidase.

\subsection{JC-1 Staining}

The fluorescent dye JC-1 (5,5'6,6'-tetrachloro-1,1,3,3'-tetraethylbenzimidazolcarbocyanine iodide, Molecular Probes, USA) was used to measure the activity of mitochondria. A stock solution of JC-1 was made at a concentration of $1 \mathrm{mg} / \mathrm{ml}$ in dimethyl sulfoxide. Fresh staining solution $(10 \mu \mathrm{g} / \mathrm{ml})$ was prepared by diluting the stock solution in warm $\left(37^{\circ} \mathrm{C}\right)$ culture medium supplemented with $10 \%$ calf serum. For each 10 oocytes, $50 \mu 1$ of fresh JC-1 solution was immediately applied to a slide. They were then placed in an incubator for $30 \mathrm{~min}$. A Partec CyScope fluorescence microscope was used for all experiments. An Argon laser was used to produce the excitation laser line at $472 \mathrm{~nm}$, and emission wavelengths were separated by a $500 \mathrm{~nm}$ dichroic mirror followed by analysis in a photomultiplier after further filtering through a $536 \mathrm{~nm}$ band pass filter (green emission). Images were processed by using Videology and Image-J software.

\subsection{Avidin-FITC Staining}

Fluorescent assessment of avidin-FITC was used for detection of 8-OHdG (Struthers, Patel, Clark, \& Thomas, 1998). The denuded oocytes were washed in PBS after fixing with $2 \%(\mathrm{w} / \mathrm{v})$ paraformaldehyde (in PBS at $\mathrm{pH} 7.4$ ) for $15 \mathrm{~min}$. Then, oocytes were washed in warm PBS twice. They were later fixed and permeabilized with ice-cold methanol for $15 \mathrm{~min}$. They were washed twice with PBS and then the oocytes were chemically blocked for $10 \mathrm{~min}$ at room temperature in $300 \mu \mathrm{l}$ PBS containing 10\% (w/v) NGS (normal goat serum). The blocking solution was washed off with PBS containing $0.2 \%$ (w/v) NGS. For each 10 oocytes, $50 \mu 1$ of avidin-conjugated FITC solution (suspended 1:200 in PBS) was immediately applied to a slide, which was then incubated for an hour at room temperature. A Partec CyScope fluorescence microscope was used for all experiments. An Argon laser was used to produce the excitation laser line at $470 \mathrm{~nm}$, and emission wavelengths were separated by a $500 \mathrm{~nm}$ dichroic mirror followed by analysis in a photomultiplier after further filtering through a $550 \mathrm{~nm}$ band pass filter. Images were processed by the Videology and fluorescence intensities were analysed by the Image-J software (Burgess et al., 2010; Gavet \& Pines, 2010; Potapova, Sivakumar, Flynn, Li, \& Gorbsky, 2011).

\subsection{Experimental Design}

Experiment 1: COCs and oocyte size measurement.

COCs and oocyte diameter was measured using Image-J software after the images were processed by Videology for CyScope microscope connected to an USB CCD camera. A mean of two measurements for each COCs and oocyte diameter was made perpendicular to each other.

Experiment 2: Determination of optimum glucose and pyruvate concentration for mitochondrial organization in matured oocytes.

A minimum of 60 oocytes were matured in each group of IVM media with four different glucose concentrations 0 , 1.5, 5.6 and $20.0 \mathrm{mM}$, respectively (modification of Hashimoto, Minami, Yamada, \& Imai, 2000) and four different sodium pyruvate concentrations $0,0.1,0.2$ and $0.4 \mathrm{mM}$. Each group had undergone an incubation period and stained with JC-1 fluorescent probes. Mitochondrial organization pattern and activity in matured oocytes were factored-in during statistical analysis.

Experiment 3: Determination of optimum glucose and pyruvate concentration for oxidative stress level in matured oocytes.

A minimum of 60 oocytes were matured in each group of IVM media with four different glucose concentrations 0 , 1.5, 5.6 and 20.0 mM, (modification of Hashimoto, Minami, Yamada, \& Imai, 2000) and four different sodium pyruvate concentrations $0,0.1,0.2$ and $0.4 \mathrm{mM}$. Each group had undergone an incubation period and stained with avidin-FITC fluorescent probes. Corrected total cell fluorescence (CTCF) was used to determine the level of fluorescence in a given region (oocyte) and this fluorescence intensity expressed the oxidative stress level. The method of calculation is, CTCF $=$ Integrated density $-($ Area of selected cell $\times$ Mean fluorescence of background readings) (Burgess et al., 2010; Gavet \& Pines 2010; (Burgess et al., 2010; Gavet \& Pines, 2010; Potapova, Sivakumar, Flynn, Li, \& Gorbsky, 2011).

\subsection{Statistical Analysis}

All oocytes were randomly distributed within each experimental group and each experiment was repeated at least three times. All percentage data were subjected to arc-sine transformation before statistical analysis. Statistical analyses were carried out using Pearson Correlation test and two-way analysis of variance (ANOVA). For the 
statistical analysis, IBM SPSS Statistics 19 software was used. Data were expressed as mean \pm S.E.M. Differences of $\mathrm{P}<0.05$ were considered significant.

\section{Results}

Experiment 1: Quality assessments of bovine oocytes (maturation) are shown in Figure 1. Our results had indicated that there was a strong relationship between oocyte diameter and the outer diameter of zona pellucida. There was a strong positive relationship in which the oocyte diameter had a $53.1 \%$ effect on the outer diameter of zona pellucida. The relationship between the two factors are expressed linearly as: Outer diameter of zona pellucida $=$ [Oocyte diameter] $\times 0.73+63.373$. We conclude that oocyte diameter or outer diameter of zona pellucida can be used to determine oocyte maturation to a certain degree. Despite that, we would like to note that other factors have played a role in determining the actual size of the zona pellucida. It would be unethical to conclude that oocyte diameter is a true reflection of the dimension of COCs. Further research should be done to identify factors that contribute to the diameter of COCs and eventually how far along the oocyte had matured.
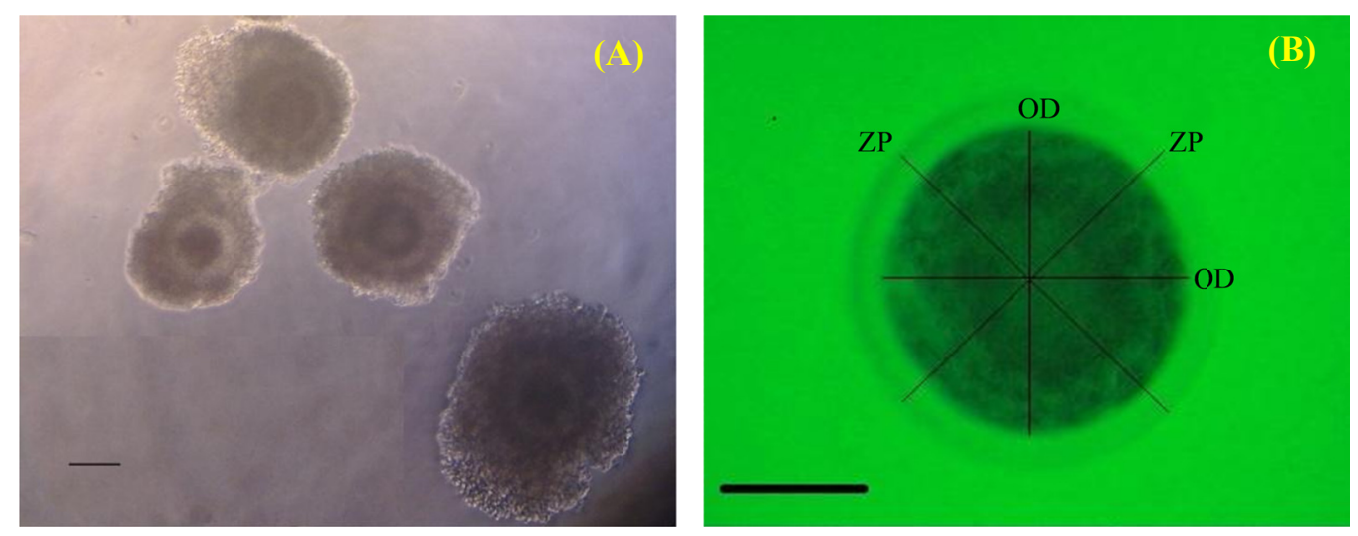

Figure 1. Quality assessments of bovine oocytes performed by the following methods: (A) Morphology of the cumulus-oocyte-complex (COCs); (B) Size determination

Note. $\mathrm{OD}=$ oocyte diameter, $\mathrm{ZP}=$ outer diameter of zona pellucida. Scale bar: for $\mathrm{A}=100 \mu \mathrm{m}$; for $\mathrm{B}=50 \mu \mathrm{m}$.

The effects of different concentrations of pyruvate and glucose in the maturation medium on diameter of bovine oocytes are shown in Table 1. There is significant difference of oocytes diameter of glucose concentrations (1.5, 5.6 and $20.0 \mathrm{mM}$ ) compared to control. No significant difference was observed in oocytes diameter of pyruvate concentrations $(0.1,0.2$ and $0.4 \mathrm{mM})$ compared to control.

Table 1. Effect of pyruvate and glucose in the maturation medium on diameter of bovine oocytes

\begin{tabular}{|c|c|c|c|c|c|c|c|}
\hline \multirow{2}{*}{$\begin{array}{l}\text { Treatment } \\
\text { Concentration (mM) }\end{array}$} & \multirow{2}{*}{$\begin{array}{l}\text { Control } \\
0\end{array}$} & \multicolumn{3}{|c|}{ Pyruvate } & \multicolumn{3}{|c|}{ Glucose } \\
\hline & & 0.1 & 0.2 & 0.4 & 1.5 & 5.6 & 20.0 \\
\hline Number of oocytes examined (n) & 60 & 62 & 86 & 77 & 63 & 70 & 76 \\
\hline Oocytes Diameter $(\mu \mathrm{m})$ & $121.37 \pm 1.09^{\mathrm{a}}$ & $124.6 \pm 9.31$ & $124.01 \pm 10.67$ & $122.71 \pm 8.4$ & $113.52 \pm 12.43^{b}$ & $111.14 \pm 14.06^{\mathrm{b}}$ & $106.16 \pm 9.23^{b}$ \\
\hline
\end{tabular}

Note. Three replicate trials were conducted. Values are expressed as mean \pm SEM. ${ }^{\mathrm{b}}$ indicates significantly different $(\mathrm{p}<0.05)$ compared to ${ }^{\mathrm{a}}$.

Experiment 2: Mitochondrial aggregation patterns and activity during oocyte maturation are shown in Figure 2. In germinal vesicle breakdown stage, the mitochondria are seen concentrated in clusters surrounding the nucleus. They are granular with a definite clump aggregation. As they mature and reach metaphase I, the mitochondrial tend to migrate and distribute to the peripheral of the oocytes. During this time the mitochondrial looks smooth to touch. Metaphase II stage mitochondria were seen aggregating around the meiotic spindle and at the oocyte centre. At this stage, oocyte had a smooth and moderately granulated cytoplasm. An intact polar body was also noted (Wilding et al., 2001; Vellila, Gonzalez, Vidal, Izquierdo, \& Panamio, 2006; Ramalho-Santos et al., 2009). 

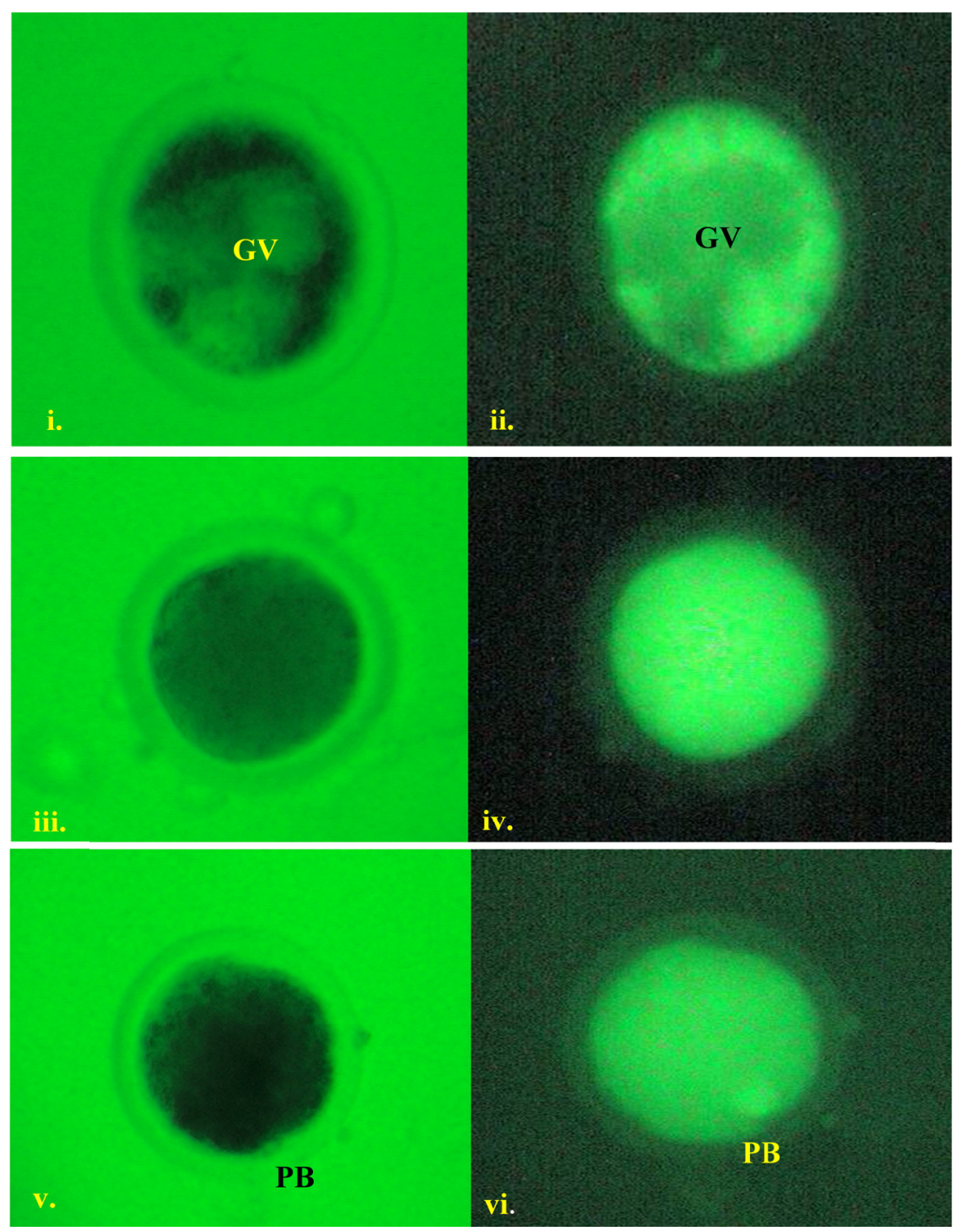

Figure 2. Mitochondrial aggregation patterns and activity during oocyte maturation

Note. Image on the left is transmitted light image, image on the right is fluorescence image taken simultaneously. (i, ii) images of germinal vesicle stage oocytes representatives of a total of 268; (iii, iv) images from metaphase I-stages oocytes representatives of a total of 169 ; (v, vi) images of metaphase II-stages oocytes representatives of a total of $57 . \mathrm{GV}=$ germinal vesicle; $\mathrm{PB}=$ polar body.

The effect of oocytes diameter at a specific stage of mitochondrial organization using $\mathrm{JC} 1$ is shown in Table 2. We would like to note that metaphase II stage oocytes had mean diameter of $124.75 \pm 1.5 \mu \mathrm{m}$. Thus, we conclude that an oocyte with a diameter larger than $120 \mu \mathrm{m}$ is fully competent.

Table 2. Effect of bovine oocytes diameter at a specific stage of mitochondrial organization using JC1

\begin{tabular}{ll}
\hline Specific stages of mitochondrial organization using JC1 & Oocyte diameter $(\boldsymbol{\mu m})$ \\
\hline Germinal vesicle breakdown (GVBD) & $268^{\mathrm{c}}(115.09 \pm 0.8)$ \\
Metaphase I (MI) & $169^{\mathrm{c}}(119.33 \pm 0.8)$ \\
Metaphase II (MII) & $57^{\mathrm{c}}(124.75 \pm 1.5)$ \\
\hline
\end{tabular}

Note. Three replicate trials were conducted. Values are expressed as mean \pm SEM. ${ }^{c}$ indicates number of oocyte examined. 
The energy substrate concentrations for each of the intervention groups based on mitochondrial organization are shown in Figure 3. There was a significant difference of energy substrate towards oocyte maturation $\left[X^{2}(12, N=\right.$ $494)=50.225, \mathrm{p}<0.05]$. The supplementation of glucose $(5.6 \mathrm{mM})$ had significantly induced resumption of meiosis and promoted oocytes maturation to metaphase II (MII) stage much faster compared with control and glucose $(1.5 \mathrm{mM}$ and $20 \mathrm{mM})$. The incidence of oocytes reaching the MII stage was significantly increased in the presence of $0.2 \mathrm{mM}$ pyruvate. Thus, the possible optimum concentrations based on percentages of metaphase II stage are pyruvate $(0.2 \mathrm{mM})$ and glucose $(5.6 \mathrm{mM})$.

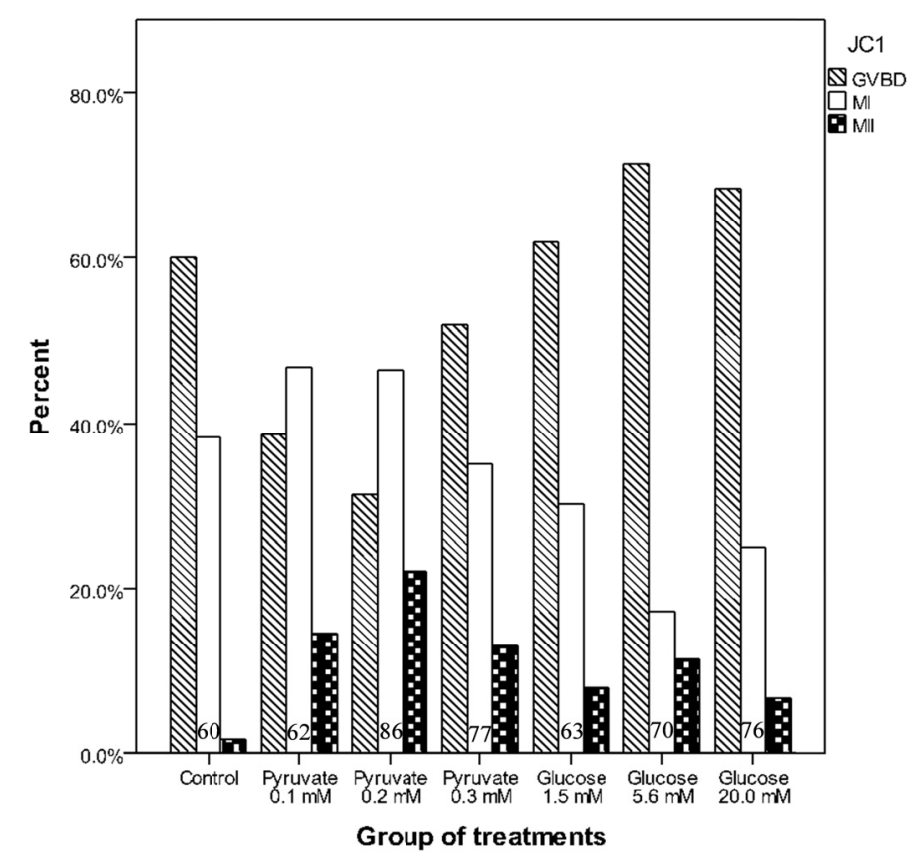

Figure 3. Energy substrate concentrations for each of the intervention groups based on mitochondrial organization Note. Three replicate trials were conducted. This figure consists of percentages of oocytes reaching GVBD = germinal vesicle breakdown stage, MI = metaphase I-stage, MII = metaphase II-stage for each of intervention groups. The number within each bar represents the total number of oocytes observed for each treatment.

Experiment 3: The effect of different concentrations of glucose and pyruvate for oxidative stress level in matured oocytes are shown in Figure 4. Corrected total cell fluorescence (CTCF) was used to determine the fluorescence intensity of oxidative stress level in bovine oocytes. There was a significant difference of CTCF between intervention groups of energy substrate, $F(6,439)=6.822, p<0.05$. The supplementation of pyruvate $(0.1,0.2$ $\mathrm{mM})$ and glucose $(1.5,5.6,20.0 \mathrm{mM})$ significantly reduced the oxidative stress level compared with control. 


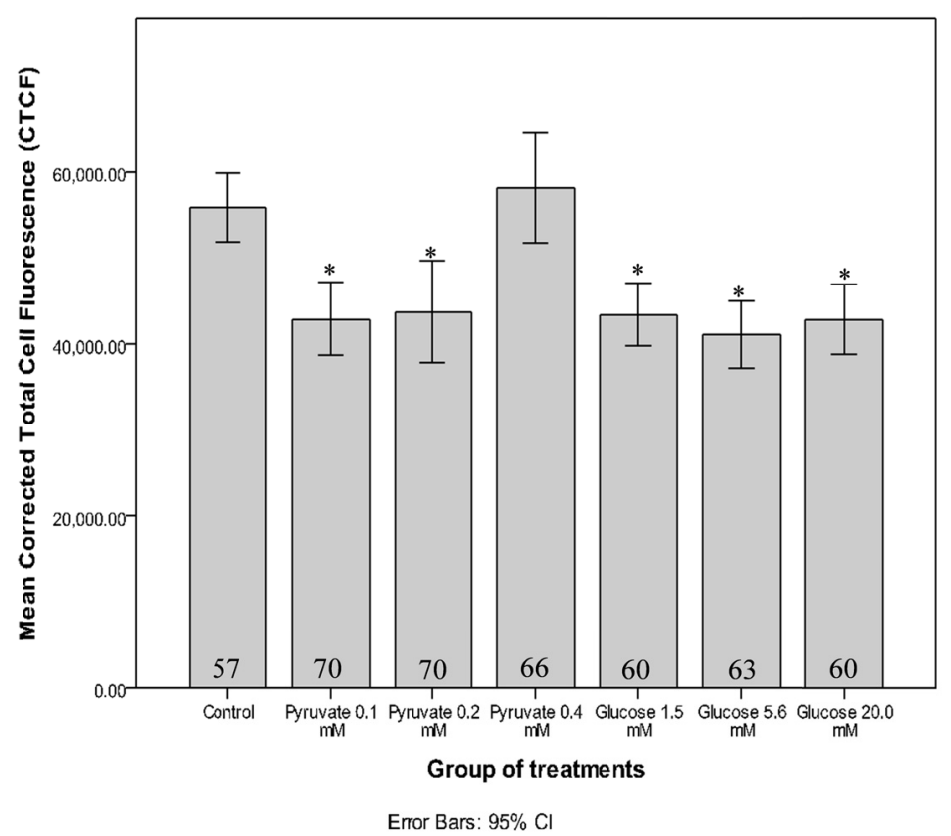

Figure 4. Energy substrate concentrations for each of the intervention groups based on oxidative cell's stress level Note. Mean of corrected total cell fluorescence (CTCF) expressed the oxidative cell's stress level. Three replicate trials were conducted. The number within each bar represents the number of oocytes observed for each treatment. Values are expressed as mean \pm SEM. Value * indicate a significantly different compared to control, $\mathrm{p}<0.05$.

\section{Discussions}

The quality of oocytes is a complex feature and not easily evaluated by non-invasive means. COCs morphology which is routinely used for evaluating oocyte quality is very subjective and seemingly does not fully predict oocyte competence (Yavari et al., 2007; Pawlak et al., 2011). Thus, the present study attempted to assess oocyte quality based on size determination and metabolic activity.

COCs diameter were first measured before and after IVM, and then correlated with diameter of denuded oocytes. Unfortunately, the present study found a poor relationship between COCs diameter and oocyte diameter. Hence, this result rejected our hypothesis that COCs diameter was a good reflection of oocyte maturation.

In the present study, supplementation of maturation medium with pyruvate had not significantly affected the diameter of the oocytes. Interestingly, the present study showed that oocyte has reach metaphase II when the diameter exceeds $120 \mu \mathrm{m}$. This stage is a mature stage. This confirms the previous findings of Pawlak, Renska, Pers-Kamczyc, Warzych, and Lechniak (2011), and Hunter (2000) which had demonstrated that most oocytes with a diameter $\geq 120 \mu \mathrm{m}$ were considered matured and fully competent. Nevertheless, we had noted that by adding higher concentrations of glucose in maturation medium, oocyte had shrunk and thus smaller. This would have indicated that minimum diameter of matured oocyte in maturation medium of glucose is $115 \mu \mathrm{m}$ and not $120 \mu \mathrm{m}$ as previously indicated. The shrinking effect could be due to the osmosis effect glucose onto the cell (oocyte). As the concentration of glucose increases, the rate of osmosis increases so the oocyte will lose more water. This is translated into the mass and diameter of the oocyte. This finding is supported by Chang, Dale, and Moley (2005), reported that oocytes derived from mice with type I diabetes with hyperglycaemia are $\sim 30 \%$ smaller in size compared to oocytes from control (Chang et al., 2005). Further studies would need to be conducted to determine if the small size oocyte have similar fertilisation capacity compared to normal oocytes.

The present study had also clearly demonstrated that the addition of pyruvate $(0.2 \mathrm{mM})$ to the maturation medium had increased the rate of maturation in bovine oocytes. This finding is in line with Deldar et al. (2011) who demonstrated that by increasing the pyruvate concentration from 0.1 to $0.33 \mathrm{mM}$ in the maturation medium, maturation rate of oocyte had increased significantly. Our results had also revealed that the supplementation of glucose $(5.6 \mathrm{mM})$ had enhanced the incidence of bovine oocytes reaching metaphase II stage. However, supplementation of low glucose $(<2.3 \mathrm{mM})$ or high concentration of glucose $(>10 \mathrm{mM})$ were detrimental to oocyte development (Sutton-McDowall, Gilchrist, \& Thompson, 2005; Sutton-McDowall, Gilchrist, \& Thompson, 
2010; Deldar et al., 2011). In this present study, a similar trend of development of glucose was observed. By culturing bovine oocyte (COCs) in medium containing lower concentration $(1.5 \mathrm{mM})$ or higher concentration $(20$ $\mathrm{mM}$ ) of glucose, the resumption of meiosis and completion of maturation to metaphase-II stage was vastly reduced. Similar pattern was also observed by Chang, Dale, and Moley (2005) which had reported that maternal diabetes and hyperglycaemia have adversely affected oocyte maturation (Chang et al., 2005). This had led to poor developmental competence.

The present study demonstrated that supplementation of pyruvate $0.1 \mathrm{mM}$ and $0.2 \mathrm{mM}$ in maturation medium significantly reduced the oxidative cell's stress level in oocytes. Addition of $0.4 \mathrm{mM}$ pyruvate in maturation medium had not significantly reduced the oxidative cell's stress level in oocytes compared to control. This results supported recent observation that pyruvate had a bi-functional role both as an energy substrate and as an antioxidant (Nandi, Kumar, Manjunatha, Ramesh, \& Gupta, 2008). Hashimoto, Minami, Yamada, and Imai (2000) reported that glucose during oocyte maturation had increased the contents of reactive oxygen species in oocytes, decreased glutathione levels and impaired the developmental competence of oocytes. Furthermore, previous studies have suggested that the toxic effect of ROS generated had caused a negative feedback of cell respiration and disruption of mitochondrial organization. All this had resulted in precocious resumption of nuclear maturation (Barnett, Clayton, Kimura, \& Bavister, 1997; Sutton-McDowall, Gilchrist, \& Thompson, 2010). Conversely, present study found that addition of glucose in maturation medium had significantly reduced the oxidative cell's stress level in oocytes compared to control maturation medium.

Taken together, these data suggest that supplementation of $0.2 \mathrm{mM}$ pyruvate in maturation medium of bovine oocytes will produce matured oocytes with a diameter $\geq 120 \mu \mathrm{m}$. Moreover, the oocyte will not shrink as observed with the addition of glucose. Resumption of meiosis will also occur thus allowing the oocytes to mature to metaphase II (MII) stage much faster. Oocytes supplemented with pyruvate recorded lower oxidative stress levels.

On the other hand, the addition of $5.6 \mathrm{mM}$ glucose in the maturation media would only enhance the oocyte maturation rate but not deliver other additional benefits as done by pyruvate. Further understanding on glucose metabolism during oocyte maturation may lead to improved IVM culture conditions, as well as an involvement in planning of treatment for diabetic or obese women with low fertility (Pasquali et al., 2007).

In conclusion, results obtained in the present study demonstrated that $0.2 \mathrm{mM}$ pyruvate is the optimum concentration for mitochondrial organisation while taking into consideration oxidative stress levels of matured bovine oocyte.

\section{References}

Anguita, B., Jimenez, A. R., Izquierdo, D., Mogas, T., \& Paramio, M. T. (2007). Effect of oocyte diameter on meiotic competence, embryo development, P34 (cdc2) expression and MPF activity in prepubertal goat oocytes. Theriogenology, 67(3), 526-536. https://doi.org/10.1016/j.theriogenology.2006.09.003

Barnett, D. K., Clayton, M. K., Kimura, J., \& Bavister, B. D. (1997). Glucose and phosphate toxicity in hamster preimplantation embryos involves disruption of cellular organization, including distribution of active mitochondria. Molecular Reproduction and Development, 48, 227-237. https://doi.org/10.1002/ (SICI)1098-2795(199710)48:2<227: AID-MRD10>3.0.CO;2-V

Biggers, J. D., Whittingham, D. G., \& Donahue, R. P. (1967). The pattern of energy metabolism in the mouse oocyte and zygote. Zoology, 58(2), 560-567. https://doi.org/10.1073/pnas.58.2.560

Blerkom, J. V., \& Davis, P. (2007). Mitochondrial signalling and fertilization. Molecular Human Reproduction, 13(11), 759-770. https://doi.org/10.1093/molehr/gam068

Burgess, A., Vigneron, S., Brioudes, E., Labbe, J.-C., Lorca, T., \& Castro, A. (2010). Loss of human great wall results in G2 arrest and multiple mitotic defects due to deregulation of the cyclin B-Cdc2/PP2A balance. Proceeding of the National Academy of Sciences of USA, 107(28), 12564-12569. https://doi.org/10.1073/pnas.0914191107

Chang, A. S., Dale, A. N., \& Moley, K. H. (2005). Maternal diabetes adversely affects preovulatory oocyte maturation, development, and granulosa cell apoptosis. Endocrinology, 146(5), 2445-2453. https://doi.org/10.1210/en.2004-1472

Deldar, H., Zare-Shahneh, A., Zamiri, M. J., Daliri, M., Kohram, H., Ansari-Pirsaraie, Z., ... Khodaie, M. (2011). Effect of glucose, lactate and pyruvate concentrations on in vitro growth of goat granulosa cell. African Journal of Biotechnology, 10(40), 7874-7877. https://doi.org/10.5897/AJB11.828 
Ebner, T., Moser, M., Sommergruber, M., \& Tews, G. (2003). Selection based on morphological assessment of oocytes and embryos at different stages of preimplantation development. Human Reproduction, 9(3), 251-262. https://doi.org/10.1093/humupd/dmg021

Gavet, O., \& Pines, J. (2010). Progressive activation of Cyclin B1-Cdk1 coordinates entry to mitosis. Development Cell, 18(4), 533-543. https://doi.org/10.1016/j.devcel.2010.02.013

Han, Z. B., Lan, G. C., Wu, Y. G., Han, D., Feng, W. G., Wang, J. Z., \& Tan, J. H. (2006). Interactive effects of granulosa cell apoptosis, follicle size, cumulus-oocyte complex morphology, and cumulus expansion on the developmental competence of goat oocytes: a study using the well-in-drop culture system. Reproduction, 132, 749-758. https://doi.org/10.1530/REP-06-0055

Hashimoto, S., Minami, N., Yamada, M., \& Imai, H. (2000). Excessive concentration of glucose during in vitro maturation impairs the developmental competence of bovine oocytes after in vitro fertilization: relevance to intracellular reactive oxygen species and glutathione contents. Molecular Reproduction and Development, 56(4), 520-526. https://doi.org/10.1002/1098-2795(200008)56:4<520::AID-MRD10>3.0.CO;2-0

Herrick, J. R., Lane, M., Gardner, D. K., Behboodi, E., Memili, E., Balash, S., ... Krisher, R. L. (2006). Metabolism, protein content and in vitro embryonic development of goat cumulus-oocyte complexes matured with physiological concentrations of glucose and L-lactate. Molecular Reproduction and Development, 73(2), 255-266. https://doi.org/10.1002/mrd.20407

Hunter, M. G. (2000). Oocyte maturation and ovum quality in pigs. Reviews of Reproduction, 5, $122-130$. https://doi.org/10.1530/ror.0.0050122

Ishizaki, C., Watanabe, H., Bhuiyan, M. M. U., \& Fukui, Y. (2009). Developmental competence of porcine oocytes selected by brilliant cresyl blue and matured individually in a chemically defined culture medium. Theriogenology, 72(1), 72-80. https://doi.org/10.1016/j.theriogenology.2009.02.015

Krisher, R. L. (2004). The effect of oocyte quality on development. Journal of Animal Science, 82(13), 14-23. https://doi.org/10.2527/2004.8213_supplE14x

Nandi, S., Kumar, V. G., Manjunatha, B. M., Ramesh, H. S., \& Gupta, P. S. P. (2008). Follicular fluid concentrations of glucose, lactate and pyruvate in buffalo and sheep, and their effects on cultured oocytes, granulosa and cumulus cells. Theriogenology, 69(2), 186-196. https://doi.org/10.1016/j.theriogenology. 2007.08.036

Pasquali, R., Patton, L., \& Gambineri, A. (2007). Obesity and infertility. Current Opinion in Endocrinology, Diabetes and Obesity, 14(6), 482-487. https://doi.org/10.1097/MED.0b013e3282f1d6cb

Pawlak, P., Renska, N., Pers-Kamczyc, E., Warzych, E., \& Lechniak, D. (2011). The quality of porcine oocytes is affected by sexual maturity of the donor gilt. Reproductive Biology, 11(1), 1-18. https://oi.org/10.1016/ S1642-431X(12)60060-6

Potapova, T. A., Sivakumar, S., Flynn, J. N., Li, R., \& Gorbsky, G. J. (2011). Mitotic progression becomes irreversible in prometaphase and collapses when Well and Cdc25 are inhibited. Molecular Biology Cell, 22(8), 1191-1206. https://doi.org/10.1091/mbc.E10-07-0599

Pries, K. A. Jr., G. S., \& Gardner, D. K. (2005). Metabolic markers of developmental competence for in vitro-matured mouse oocytes. Reproduction, 130, 475-483. https://doi.org/10.1530/rep.1.00831

Ramalho-Santos, J., Varum, S. Amaral, S., Mota, P. C., Sousa, A. P., \& Amaral, A. (2009). Mitochondrial functionality in reproduction: From gonads and gametes to embryos and embryonic stem cells. Human Reproduction Update, 15(5), 553-572. https://doi.org/10.1093/humupd/dmp016

Roberts, R., Stark, J., Iatropoulou, A., Becker, D. L., Franks, S., \& Hardy, K. (2004). Energy substrate metabolism of mouse cumulus-oocyte-complexes: Response to follicle-stimulating hormone is mediated by the phosphatidylinositol 3-kinase pathway and is associated with oocyte maturation. Biology of Reproduction, 71(1), 199-209. https://doi.org/10.1095/biolreprod.103.023549

Sirad, M. A., \& Blondin, P. (1996). Oocyte maturation and IVF in cattle. Animal Reproduction Science, 42, 417-426. https://doi.org/10.1016/0378-4320(96)01518-7

Smiley, S. T., Reers, M., Mottola-Hartshorn, C., Lin, M., Chen, A., Smith, T. W., ...Chen, L. (1991). Intracellular heterogeneity in mitochondrial membrane potentials revealed by a J-aggregate forming lipophilic cation JC-1. Proceeding of the National Academy of Sciences of USA, 88(9), 3671-3675. https://doi.org/10.1073/ pnas.88.9.3671 
Struthers, L., Patel, R., Clark, J., \& Thomas, S. (1998). Direct detection of 8-oxodeoxyguanosine and 8-oxoguanine by avidin and its analogues. Analytical Biochemistry, 255(1), 20-31. https://doi.org/10.1006/ abio.1997.2354

Sugino, N. (2005). Reactive oxygen species in ovarian physiology. Reproductive Medicine \& Biology, 4(1), 31-44. https://doi.org/10.1111/j.1447-0578.2005.00086.x

Sugino, N. (2006). Roles of reactive oxygen species in the corpus luteum. Animal Science Journal, 77(6), 556-565. https://doi.org/10.1111/j.1740-0929.2006.00386.x

Sutton-McDowall, M. L., Gilchrist, R. B., \& Thompson, J. G. (2005). Effect of hexoses and gonadotropin supplementation on bovine oocyte nuclear maturation during in vitro maturation in a synthetic follicle fluid medium. Reproduction, Fertility and Development, 17(4), 407-415. https://doi.org/10.1071/RD04135

Sutton-McDowall, M. L., Gilchrist, R. B., \& Thompson, J. G. (2010). The pivotal role of glucose metabolism in determining oocyte developmental competence. Reproduction, 139, 685-695. https://doi.org/10.1530/ REP-09-0345

Tamura, H., Takasaki, A., Miwa, I., Taniguchi, K., Maekawa, R., Asada, H., ... Sugino, N. (2007). Oxidative stress impairs oocyte quality and melatonin protects oocytes from free radical damage and improves fertilization rate. Journal of Pineal Research, 44(3), 280-287. https://doi.org/10.1111/j.1600-079X.2007.00524.x

Thompson, J. G., Lane, M., \& Gilchrist, R. B. (2007). Metabolism of the bovine cumulus-oocyte complex and influence on subsequent developmental competence. Society of Reproduction and Fertility Supplement, 64, 179-190. https://doi.org/10.5661/RDR-VI-179

Tsuji, H., Lee, J. H., Hossain, M. S., Tareq, K. M. A., Hamano, K., \& Sawada, T. (2009). The beneficial effect of fructose and glucose on in vitro maturation and the fertilization of porcine oocytes. Reproductive Medicine \& Biology, 8(1), 19-24. https://doi.org/10.1007/s12522-008-0003-8

Vellila, E., Gonzalez, E., Vidal, F., Izquierdo, D., \& Panamio, M. (2006). Mitochondrial organization in prepubertal goat oocytes during in vitro maturation and fertilization. Molecular Reproduction \& Development, 73(5), 617-626. https://doi.org/10.1002/mrd.20426

Wallace, D. C. (1999). Mitochondrial disease in man and mouse. Science, 283(5407), 1482-1488. https://doi.org/ 10.1126/science.283.5407.1482

Wilding, M., Dale, B., Marino, M., Matteo, L., Alviggi, C., Pisaturo, M. L., ... Placido, G. (2001). Mitochondrial aggregation patterns and activity in human oocytes and preimplantation embryos. Human Reproduction, 16(5), 909-917. https://doi.org/10.1093/humrep/16.5.909

Yavari, M., Fujii, A., Shimizu, R., Ito, A., Kaedei, Y., Morita, Y., ... Otoi, T. (2007). Meiotic competence of porcine oocytes after percoll sedimentation treatment for oocyte selection. Journal of Animal and Veterinary Advances, 6(11), 1333-1336.

\section{Copyrights}

Copyright for this article is retained by the author(s), with first publication rights granted to the journal.

This is an open-access article distributed under the terms and conditions of the Creative Commons Attribution license (http://creativecommons.org/licenses/by/4.0/). 